\title{
Editorial
}

\section{Monitoring birth defects}

Fifty years ago, Gregg's classic observations linked congenital cataract to infection of the mother with rubella during pregnancy. It is almost exactly thirty years ago that the drama of the thalidomide shocked the world into a state of awareness as regards the potential of external, man-made influences to harm the unborn child. Society, most unhappily, has a way of neglecting major health issues until something goes drastically wrong; the tragedy of thalidomide at least served to ensure that something would be done to prevent the recurrence of anything so terrible. At least where medicines were concerned, and to some extent as regards new potential toxins coming into use in other fields, procedures were established to that end, largely directed to the performance of pre-marketing safety studies as a basis for approval.

In various respects, nevertheless, the reaction to the thalidomide disaster was inadequate. In part that was because of the belief that animal models, if properly developed, could provide a tool for identifying risks and eliminating human exposure; subsequent experience pointed all to clearly to the limitations of that approach. The problem, however, also lay in the very nature of the reaction to thalidomide; the fact that many of the defects which it induced were gross, immediately visible at birth, and present in a substantial proportion of the children exposed suggested misleadingly that analogous problems in the future would be as readily and rapidly detectable. That did not prove to be the case. The risks of diethylstilboestrol which were defined in the mid-seventies became only apparent when the offspring reached adolescence or even later during the fertile period of life; debates such as those relating to bendictin/debendox and to the possible risks of benzodiazepines illustrated the problems of identifying marginal teratogenicity; and the cases of lead and alcohol were among those which opened up the equally problematical field of behaviourial teratogenicity.

For all these reasons, the last three decades have led us increasingly in the direction of large-scale monitoring to register possible birth defects and evidence of their link to external factors. The starting assumption is that no form of pre-marketing scrutiny, however necessary, will entirely eliminate risks with new substances, let alone dangers posed by any of the many tens of thousands to which humans are currently exposed. Monitoring, on the other hand, should enable one increasingly to detect problems of current exposure, so that steps can be taken to eliminate them as early as possible.

Monitoring, as J. David Erickson recalls in this issue of the International Journal of Risk and Safety in Medicine began within a few years of the thalidomide tragedy, and within a decade it had become established in a range of countries. It is an 
activity typically demanding input from a wider range of countries; in some of these, the input provided will be primarily of quantitative importance, because they are in a position to provide data on the grand scale; in other cases the contribution is vital simply because the range of exposure to possible teratogens differs traditional and folk medicine, for example, without any doubt involve exposure to certain natural teratogens, only a few of which have so far been identified. The value of a global system, however, is more than merely the sum of its parts. It will increasingly serve to catalyze and refine the process of monitoring in each of the countries contributing to it, enabling one to benefit from both the methodological experience and the findings of another. Above all, the growth of a global system will enable early signals to be pieced together from fragments of evidence which might otherwise remain scattered.

For such reasons, the work of the International Clearinghouse for Birth Defects Monitoring Systems is vital. A compact overview of the work of the Clearinghouse and its Centre in Bergen was presented to the Annual Meeting of the European Teratology Society in Edinburgh in September 1990, and the papers read there constitute this issue of the Journal, which since its inception has devoted particular attention to the fundamental problem of pregnancy protection. Detailed information with 15 years data and evaluations of birth defect monitoring by the Clearinghouse can be found in the book Congenital Malformations Worldwide, Elsevier, 1991.

We would express our thanks to J. David Erickson, Stein Emil Vollset and Paul Peters as editors of the present issue, and to Pat Schreuder for her technical assistance in the preparation of the manuscripts.

M.N.G. Dukes University of Groningen June 1991

The International Journal of Risk and Safety in Medicine has a particular interest in matters related to the safety of medicine in pregnancy. Contributions to the journal from readers of this issue will be welcome at all times. 